\section{Detection of hand, foot and mouth disease in the Yucatan Peninsula of Mexico}

\author{
Carlos Machain-Williams, ${ }^{1}$ \\ Alma R. Dzul-Rosado, ${ }^{2}$ \\ Aarón B. Yeh-Gorocica, ${ }^{3}$ \\ Katia G. Rodriguez-Ruz, ${ }^{3}$ \\ Henry Noh-Pech, ${ }^{3}$ \\ Lourdes Talavera-Aguilar, ${ }^{1}$ \\ Ma. Isabel Salazar, ${ }^{4}$ \\ María Eugenia Castro-Mussot, ${ }^{4}$ \\ Guadalupe Reyes-Solis, ${ }^{1}$ \\ Julián E. Garcia-Rejon, ${ }^{1}$ \\ Fernando I. Puerto-Manzano, ${ }^{3}$ \\ Bradley J. Blitvich ${ }^{5}$
}

'Laboratorio de Arbovirología, Centro de Investigaciones Regionales Dr. Hideyo

Noguchi, Universidad Autónoma de Yucatán, México; ${ }^{2}$ Private Practice,

Mérida, Yucatán, México; ${ }^{3}$ Laboratorio de

Enfermedades Emergentes y Re-emer-

gentes, Centro de Investigaciones

Regionales Dr. Hideyo Noguchi,

Universidad Autónoma de Yucatán,

Mérida, Yucatán, México; ${ }^{4}$ Laboratorio de

Inmunología Celular e

Inmunopatogénesis, Departamento de

Inmunología, Escuela Nacional de

Ciencias Biológicas, Instituto Politécnico

Nacional, Mexico; ${ }^{5}$ Department of

Veterinary Microbiology and Preventive

Medicine, College of Veterinary Medicine,

lowa State University, Ames, IA, USA

\section{Abstract}

We report a case of hand, foot and mouth disease (HFMD) in a 5-year-old male from Merida City in the Yucatan Peninsula of Mexico. A clinical and physical examination revealed that the patient had symptoms typical of HFMD, including fever, fatigue, odynophagia, throat edema, hyperemia, lesions on the hands and feet, and blisters in the oral cavity. The patient fully recovered after a convalescence period of almost three weeks. Reverse transcription-polymerase chain reaction and nucleotide sequencing revealed that the etiological agent was enterovirus 71 (EV71). The sequence has greatest $(90.4 \%)$ nucleotide identity to the corresponding regions of EV71 isolates from the Netherlands and Singapore. Although HFMD is presumably common in Mexico, surprisingly there are no data in the PubMed database to support this. This case report provides the first peer-reviewed evidence of HFMD in Mexico.

\section{Introduction}

Hand, foot, and mouth disease (HFMD) is a common childhood illness characterized by fever, malaise and vesicular eruptions on the hands, feet and oral mucosa. ${ }^{1-3}$ Complications are rare, but fatal cardiopulmonary and neurologic complications may occur. The most common etiological agents are enterovirus 71 (EV71) and coxsackievirus A16 (CV-A16) although other viruses in the genus Enterovirus, such as coxsackievirus A6 (CVA6), have also been associated with this illness. ${ }^{1-6}$ EV71 has been associated with severe and sometimes fatal neurological complications such as aseptic meningitis, acute flaccid paralysis, encephalitis and neurogenic pulmonary edema. HFMD is spread from person to person by contact with saliva, respiratory secretions, fluid in vesicles and feces. There are no vaccines or specific treatments available for HFMD. 7,8

Hand, foot, and mouth disease was first reported in Canada in 1957, ${ }^{9}$ and has since been detected throughout most of the world including Africa, Asia, Australia, Brazil, Europe and the United States. ${ }^{1-3}$ Historically, HFMD has been associated with sporadic cases and small outbreaks; however, in the last 15 years, there has been an alarming increase in the size, frequency and severity of HFMD outbreaks in Southeast Asia. ${ }^{5,6,10-14}$

Information on HFMD in Mexico is limited. Anecdotal evidence suggests that HFMD occurs in Mexico; for instance, ProMed was recently notified through its social media Facebook page of several apparent cases of HFMD in Mexico City (ProMed, Archive No. 20120511.1130367). However, to the best of our knowledge, there are no articles in the PubMed database that report the presence of HFMD in Mexico. Due to this gap in our knowledge, we set out to provide evidence that HFMD does indeed occur in Mexico.

\section{Case Report}

We describe a case of HFMD in a 5-year-old male (weight: $18 \mathrm{~kg}$, height: $112 \mathrm{~cm}$ ) from Merida City in the Yucatan Peninsula of Mexico. The patient first presented at a private clinic in March 2014. The patient developed mild flu-like symptoms approximately two weeks before the initial consultation. The patient's mother reported that he had interacted with an older relative with similar clinical manifestations about one week prior to illness onset. The physician was also informed that the patient had not traveled outside the state during the preceding 12 months.

A clinical and physical examination revealed
Correspondence: Bradley J. Blitvich, Department of Veterinary Microbiology and Preventive Medicine, College of Veterinary Medicine, Iowa State University, Ames, IA 50011, USA.

Tel.: +1.515.294.9861 - Fax: +1.515.294.8500.

E-mail: blitvich@iastate.edu

Key words: hand, foot and mouth disease, enterovirus, Mexico, case report.

Contributions: the authors contributed equally

Conflict of interests: the authors declare no potential conflict of interests.

Funding: this study was supported by a grant from CONACyT-FOMIX.

Received for publication: 9 September 2014. Revision received: 1 December 2014.

Accepted for publication: 3 December 2014.

This work is licensed under a Creative Commons Attribution NonCommercial 3.0 License (CC BYNC 3.0).

(O)Copyright C. Machain-Williamset al., 2014 Licensee PAGEPress, Italy

Infectious Disease Reports 2014; 6:5627

doi:10.4081/idr.2014.5627

that the patient had a fever $\left(38.0^{\circ} \mathrm{C}\right)$, generalized rash, fatigue, odynophagia, throat edema, hyperemia, clear lesions on the hands and soles of the feet, and blisters in the mouth mucosa and soft pallet (Figure 1). Lesions were also observed on the knees and genital area. A chest inspection revealed tachycardia in response to the fever. A pulmonary auscultation revealed that the patient also had a dry cough due to a mild respiratory infection with a slight increment in fremitus, but not sibilant rales, detected during inhalation and expiration. Hypoventilation in the pulmonary base was ruled out and no signs of dyspnea were observed. An abdominal scrutiny revealed no pain following superficial or deep palpation but generalized bloating was observed. A saliva sample was collected and placed at $-20^{\circ} \mathrm{C}$.

The child was sent home with a support regime of hydration, a soft diet and rest. Complementary palliative care was given and consisted of nimesulide (45 mg every 12 hours) and metisoprinol (250 $\mathrm{mg}$ of every 8 hours) per os for 5 days each. Drug dosages were based on the Vademecum medicus. The physician performed a follow-up examination once the patient had completed the treatment regimen. The patient no longer complained of symptoms. The physician noted that the mouth blisters and all lesions had disappeared with the exception of several mild dried scars.

To determine whether the patient had acquired HFMD as a result of an enterovirus 
infection, an aliquot $(100 \mu \mathrm{L})$ of the saliva sample was added to $500 \mu \mathrm{L}$ of TRIzol reagent (Invitrogen, Carlsbad, CA, USA) and total RNA was extracted according to the manufacturer's recommendations. Complementary DNAs were generated using the ImProm-II ${ }^{\mathrm{TM}}$ Reverse Transcription System with random hexamer primers (Promega, Madison, WI, USA). PCRs were performed using $T a q$ polymerase (Invitrogen) and enterovirus-specific primers, denoted as primer 1 (5'-CAAGCACTTCTGTTTCCCCGG-3') and primer 3 (5'-ATTGTCACCATAAGCAGCCA-3'), which amplify a highly conserved 440-nt region of the 5', untranslated region. ${ }^{15}$ PCR amplifications were conducted by using the following reaction conditions: $94^{\circ} \mathrm{C}$ for $3 \mathrm{~min} ; 40$ cycles of $94^{\circ} \mathrm{C}$ for $1 \mathrm{~min}, 42^{\circ} \mathrm{C}$ for $1 \mathrm{~min}$, and $72^{\circ} \mathrm{C}$ for $2 \mathrm{~min}$; followed by a final extension at $72^{\circ} \mathrm{C}$ for $10 \mathrm{~min}$. RT-PCRs performed with diethyl pyrocarbonate-treated distilled water in place of nucleic acid were included as negative controls. All test and control reactions were performed in duplicate. PCR products were examined by $2 \%$ agarose gel electrophoresis, visualized with ethidium bromide and extracted using the QIAquick gel extraction purification kit (Qiagen, Valencia, CA, USA). Purified DNAs were sequenced in both directions using an ABIprism DNA sequencer at the Instituto de Biotecnología in the National Mexican University, Mexico City, Mexico.

PCR products of the expected size were observed when the saliva sample was tested by RT-PCR using enterovirus-specific primers. Products were not observed in any of the negative control assays. The PCR products were sequenced (Genbank Accession No. KP $218059)$ and shown to have greatest $(90.4 \%)$ nucleotide identity to the corresponding regions of EV71 isolates from the Netherlands in 2007 and Singapore in 2008. The next closest matches were to the corresponding regions of EV71 isolates from Hong Kong in 2008 (90.1\%), Canada in 2007 (89.8\%), the United Kingdom in 2006 (89.8\%) and France in 2009 (89.6\%).

\section{Discussion}

We describe the clinical presentations of a laboratory-confirmed case of HFMD in a 5year-old male in Merida City in the Yucatan Peninsula of Mexico. The patient displayed symptoms characteristic of HFMD (i.e. blisters in the oral cavity and lesions on the hands and feet) and, after a convalescent period of almost three weeks, made a complete recovery. The etiological agent was identified as EV71.

We consider it likely that HFMD commonly affects children in Mexico since it is common childhood illness in most countries, including

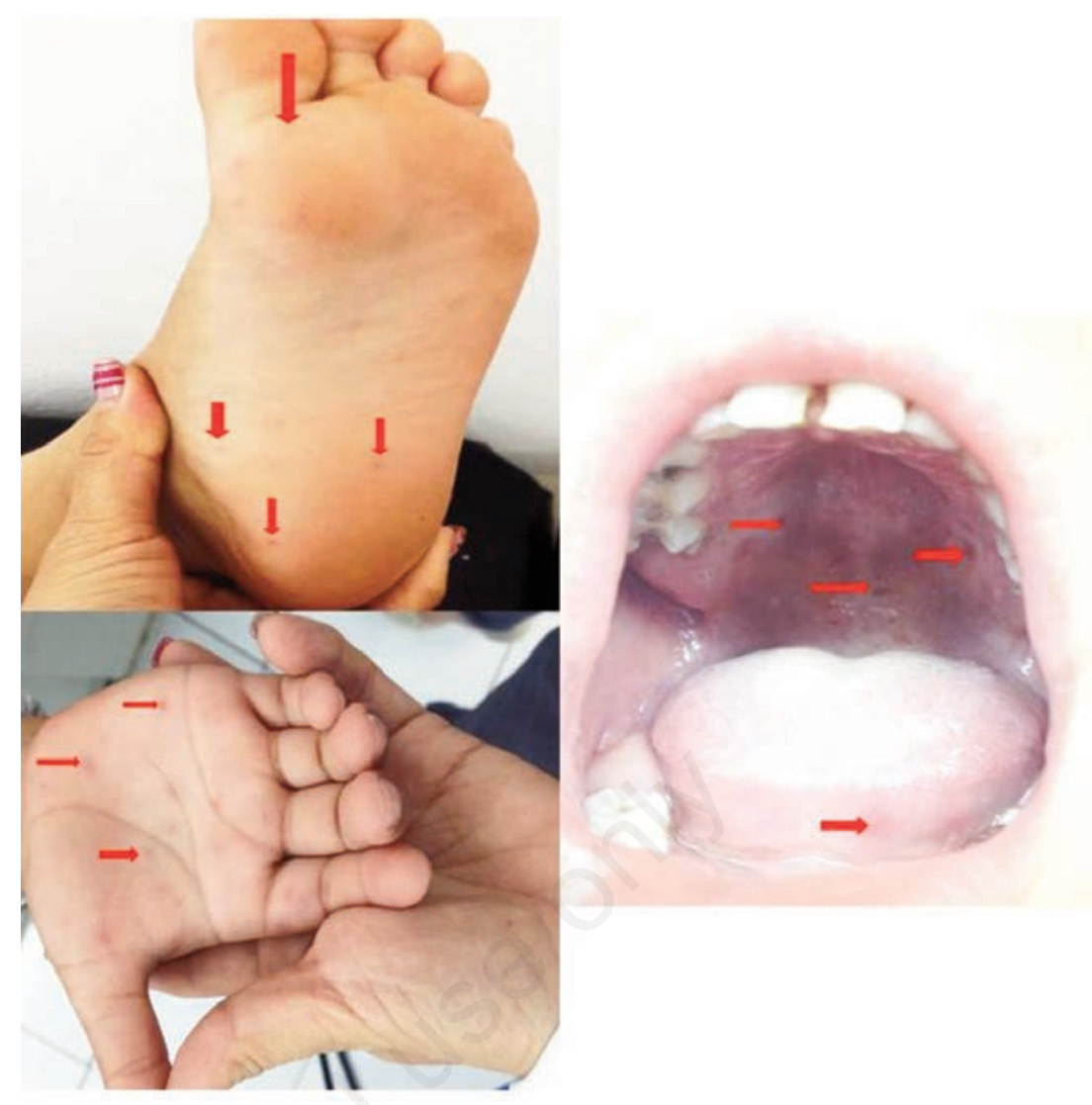

Figure 1. Lesions on the hands and soles of the feet, and blisters in the oral cavity of the patient who presented with hand, foot and mouth disease. Select lesions and blisters are indicated by red arrows.

the United States which borders Mexico to the north. ${ }^{2,16}$ Furthermore, anecdotal evidence suggests that HFMD occurs in Mexico; for example, several cases of HFMD apparently occurred among children in a daycare center in Mexico City in 2012 (ProMed, Archive No. 20120511.1130367). Nevertheless, prior to our study, there was no compelling evidence available in the public domain demonstrating that HFMD occurs in Mexico. An extensive search of the PubMed database failed to yield any articles reporting the detection of HFMD in Mexico. Likewise, a search of the Genbank database did not produce any sequences corresponding to EV71 or CV-A16 isolates from Mexico. It is also important to note that limited information was provided in the aforementioned ProMed announcement. Details on the laboratory tests (if any) that were performed were not provided and, despite a request from the ProMed moderator for additional information on the status of HFMD in Mexico, no further information was posted. Therefore, the importance of our case report cannot be understated because it provides compelling evidence that HFMD occurs in Mexico.

The EV71 sequence identified in this study exhibits a minimum of $9.6 \%$ nucleotide divergence from the corresponding regions of all other EV71 sequences in the Genbank database. A likely explanation for this considerable divergence is because our virus is spatially distinct from all other EV71 isolates listed in the Genbank database; as already noted, we report the first EV71 sequence data from Mexico. It is also interesting to note that our sequence exhibits greatest nucleotide identity to virus isolates from the Eastern Hemisphere. Similar observations were reported during an outbreak of HFMD in Cuba in 2011 to 2103; the CVA6 isolates collected during the outbreak were most closely related to isolates from Europe and Southeast Asia. ${ }^{17}$

\section{Conclusions}

Hand, foot and mouth disease is a non-notifiable disease in Mexico and this presumably is a major reason as to why there is limited information on this illness in the country. It is therefore very important that patient case studies such as this one are reported. 
Information on the clinical presentations, patient outcomes, treatment regimens and incidence of HFMD in Mexico will lead to an increased awareness of HFMD among the general public and local medical health professionals, and will result in improved EV71 screening in the pediatric community. This will in turn result in more timely diagnoses, more effective treatments and more favorable patient outcomes as well as the implementation of strategies to will reduce the spread and transmission of the disease. Improved diagnosis and treatment of EV71 is especially important given that this virus has been associated with severe and sometimes fatal neurological disease.

\section{References}

1. Wong SS, Yip CC, Lau SK, Yuen KY. Human enterovirus 71 and hand, foot and mouth disease. Epidemiol Infect 2010;138:1071-89.

2. Solomon T, Lewthwaite P, Perera D, et al. Virology, epidemiology, pathogenesis, and control of enterovirus 71. Lancet Infect Dis 2010;10:778-90.

3. Ooi MH, Wong SC, Lewthwaite P, et al. Clinical features, diagnosis, and management of enterovirus 71. Lancet Neurol 2010:1097-105.
4. Lo SH, Huang YC, Huang CG, et al. Clinical and epidemiologic features of Coxsackievirus A6 infection in children in northern Taiwan between 2004 and 2009. J Microbiol Immunol Infect 2011;44:252-7.

5. Tu PV, Thao NT, Perera D, et al. Epidemiologic and virologic investigation of hand, foot, and mouth disease, southern Vietnam, 2005. Emerg Infect Dis 2007;13:1733-41.

6. Liu W, Wu S, Xiong Y, et al. Co-circulation and genomic recombination of coxsackievirus A16 and enterovirus 71 during a large outbreak of hand, foot, and mouth disease in Central China. PloS One 2014;9:e96051.

7. Kung YA, Hung CT, Liu YC, Shih SR. Update on the development of enterovirus 71 vaccines. Expert Opin Biol Ther 2014;3:1-10.

8. Liang Z, Mao Q, Gao F, Wang J. Progress on the research and development of human enterovirus 71 (EV71) vaccines. Front Med 2013;7:111-21.

9. Robinson CR, Doane FW, Rhodes AJ. Report of an outbreak of febrile illness with pharyngeal lesions and exanthem: Toronto, summer 1957; isolation of group A Coxsackie virus. CMAJ 1958;79:615-21.

10. Sabanathan S, Tanle V, Thwaites L, et al. Enterovirus 71 related severe hand, foot and mouth disease outbreaks in SouthEast Asia: current situation and ongoing challenges. J Epidemiol Community
Health 2014;68:500-2.

11. Yip CC, Lau SK, Woo PC, Yuen KY. Human enterovirus 71 epidemics: what's next? Emerg Health Threats J 2013;6:19780.

12. Komatsu H, Shimizu Y, Takeuchi Y, et al. Outbreak of severe neurologic involvement associated with Enterovirus 71 infection. Pediatr Neurol 1999;20:17-23.

13. Ho M, Chen ER, Hsu KH, et al. An epidemic of enterovirus 71 infection in Taiwan. Taiwan Enterovirus Epidemic Working Group. N Engl J Med 1999;341:929-35.

14. Zeng M, Li YF, Wang $\mathrm{XH}$, et al. Epidemiology of hand, foot, and mouth disease in children in Shanghai 2007-2010. Epidemiol Infect 2012;140:1122-30.

15. Zoll GJ, Melchers WJ, Kopecka H, et al. General primer-mediated polymerase chain reaction for detection of enteroviruses: application for diagnostic routine and persistent infections. J Clin Microbiol 1992;30:160-5.

16. Centers for Disease Control and Prevention. Notes from the field: severe hand, foot, and mouth disease associated with coxsackievirus A6 - Alabama, Connecticut, California, and Nevada, November 2011-February 2012. MMWR Morb Mortal Wkly Rep 2012;61:213-4.

17. Fonseca MC, Sarmiento L, Resik S, et al. Coxsackievirus A6 and enterovirus 71 causing hand, foot and mouth disease in Cuba, 2011-2013. Arch Virol 2014;159:2451-5. 